Christian F. Pantoja, Y. Mauricio Muñoz-Muñoz, Lorraine Guastar, Jadran Vrabec, Julien Wist

\title{
Composition dependent transport diffusion in non-ideal mixtures from spatially resolved nuclear magnetic resonance spectroscopy
}

Journal article | Accepted manuscript (Postprint)

This version is available at https://doi.org/10.14279/depositonce-8393

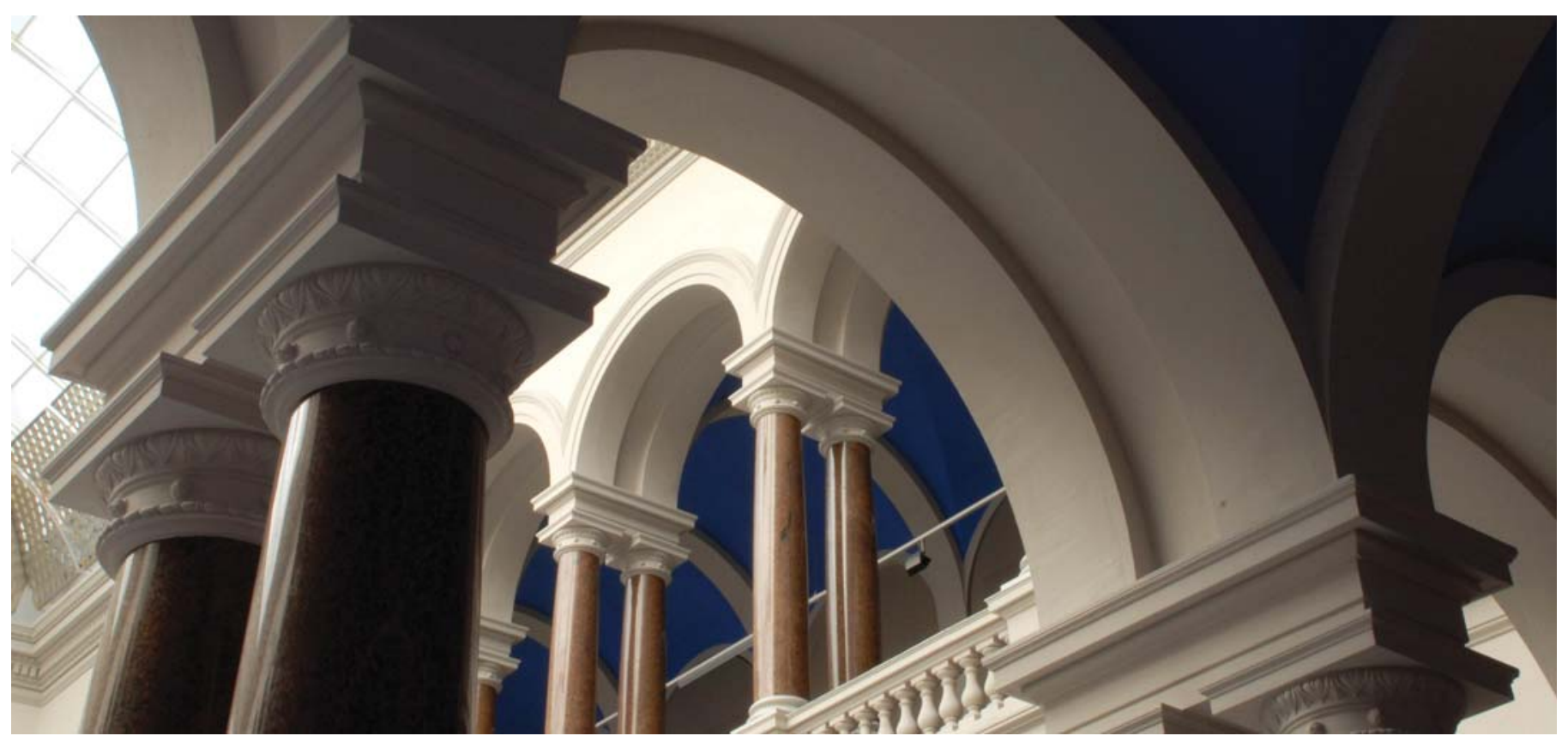

Pantoja, Christian F.; Muñoz-Muñoz, Y. Mauricio; Guastar, Lorraine; Vrabec, Jadran; Wist, Julien (2018). Composition dependent transport diffusion in non-ideal mixtures from spatially resolved nuclear magnetic resonance spectroscopy. Physical Chemistry Chemical Physics, 20(44), 28185-28192. https://doi. org/10.1039/c8cp05539d 


\title{
Composition Dependent Transport Diffusion in Non-Ideal Mixtures from Spatially Resolved Nuclear Magnetic Resonance Spectroscopy
}

\author{
Christian F. Pantoja, ${ }^{a}$ Y. Mauricio Muñoz-Muñoz ${ }^{b}$, Lorraine Guastar ${ }^{a}$, Jadran Vrabec ${ }^{b}$, and Julien \\ Wist ${ }^{* a}$ \\ Nuclear magnetic resonance (NMR) is a well-established technique for the measurement of intra-diffusion coefficients. \\ Recently, such information has been used as a basis of predictive models to extrapolate to the Fick diffusion coefficient of \\ liquid mixtures. The present work presents a new approach to directly access the Fick diffusion coefficient by spatially \\ resolved NMR experiments. The Fick diffusion coefficient of the binary mixture TEA/H2O was determined at two \\ temperatures, $283.2 \mathrm{~K}$ and $275.2 \mathrm{~K}$. The results are consistent with values previously reported either from optical \\ experiments or predictive Darken-type models developed for this system. The proposed methodology adds high-resolution \\ NMR to the toolbox for the study of transport diffusion of multicomponent mixtures. It is, however, still limited to mixtures \\ with liquid-liquid equilibrium phase separation.
}

\section{Introduction}

Transport diffusion is widely studied because of its importance to understand natural phenomena and improve industrial processes ${ }^{12}$ 3. Different experimental techniques are being employed for that task $^{4}$, typically providing diffusion coefficient data. Based on the nonetheless still surprisingly small experimental database ${ }^{5}$, a range of numerical models was proposed for their prediction ${ }^{7}$

Transport diffusion can be described by means of two formalisms, which can be transformed into each other if sufficient thermodynamic information on the system is available. The MaxwellStefan approach describes diffusion from a physically sound perspective and postulates chemical potential gradients as driving forces for mass flux ${ }^{8}{ }^{9}$. It is being used e.g. for studies of catalytic performance in reactors ${ }^{10}$, pervaporation membranes ${ }^{11}$ or for predicting transport diffusion coefficients by equilibrium molecular dynamics simulation ${ }^{12}$. Alternatively, Fick's "law" assumes concentration gradients as driving forces for mass flux, which is beneficial from a practical standpoint. The related diffusion coefficients are usually measured by Taylor dispersion ${ }^{13}$ or interferometry techniques ${ }^{14} 15$.

However, the precise measurement of diffusion coefficients entails significant experimental effort and is only possible for mixtures consisting of a small number of components (typically $\leq 3$ ). Also, most numerical predictive models explicitly focus on such systems only, mainly because of the lack of appropriate data. Consequently, the uncertainty associated with transport diffusion in multi-component mixtures is high ${ }^{16}$.

Diffusion coefficients of liquid mixtures tend to be strongly dependent on composition. In contrast to common assumptions, this holds for both Fick and Maxwell-Stefan diffusion coefficients. Experiments thus have to be carried out in a repetitive manner varying the mixture composition. An approach first proposed in the $1970 s^{17}$ has recently been revitalized by Bardow et al. ${ }^{18}$. It allows for the determination of the Fick diffusion coefficient of a liquid mixture in the entire composition range with a single experimental run if a varying spatial distribution of composition can be measured over time with a resolution that is sufficient for fitting a diffusion model to these data. A similar strategy has successfully been employed to gain insight about absorption ${ }^{19}$ as well as transport in anionic gels $\mathrm{s}^{20}$ and polyelectrolytes ${ }^{21}$. Moreover, the corresponding data can be used to discriminate models, such as in the incremental-model approach proposed by Bardow and coworkers. This model-free methodology was validated on the basis of Raman spectroscopy data ${ }^{18} 22{ }^{23}$. Because Raman spectroscopy is capable to sample the individual contribution of all components constituting a mixture, it may pave the way to the study of truly multi-component systems.

Nuclear Magnetic Resonance (NMR) spectroscopy offers comparable advantages as Raman spectroscopy. Indeed, the molar fraction of all components in a mixture can be measured with a good temporal and spatial resolution borrowing concepts from Magnetic Resonance Imaging (MRI) ${ }^{24}$. Moreover, NMR spectroscopy is suitable for the concurrent measurement of intra-diffusion coefficients, which describe the random motion of molecular species in a mixture ${ }^{25}$. It has also been used to improve model-based schemes, which rely on Raman spectroscopy measurements, especially for molar fraction regimes where the presence of small concentration gradients prohibits good estimations of transport diffusion coefficients ${ }^{26}$.

NMR spectroscopy was used in preceding work of our group to observe the temporal and spatial evolution of all molar fractions of a multi-component mixture during its mixing process, starting from a liquid-liquid equilibrium (LLE) state point ${ }^{27}$. In the present work, we propose a scheme for measuring the Fick diffusion coefficient of binary liquid mixtures by combining that experimental technique with a numerical solution of partial differential equations (PDE) for the mixing process inside a cylindrical tube. The binary mixture Triethylamine (TEA) / Water $\left(\mathrm{H}_{2} \mathrm{O}\right)$ was chosen to validate this approach. Due to its highly non-ideal thermodynamic behavior, it was necessary to employ a coordinate transformation as shown by Bardow et al. ${ }^{23}$. TEA / $\mathrm{H}_{2} \mathrm{O}$ has also recently been used to validate a predictive model by $\mathrm{D}^{\prime}$ Agostino et al. $^{28}$, which very accurately performs for its Fick diffusion coefficient over a wide composition range. Moreover, the present results are compared to experimental literature values and other recent predictive models ${ }^{29}{ }^{30}$. 


\section{Experimental}

\section{Sample preparation}

To evaluate the proposed methodology, four liquid TEA/ $\mathrm{H}_{2} \mathrm{O}$ mixture samples were prepared together with Deuterium oxide $\left(D_{2} O\right)$ purchased from Sigma-Aldrich. $\mathrm{D}_{2} \mathrm{O}$ was added for technical reasons only, i.e., to lock the resonance frequency during multiple scan acquisitions, and its amount was kept as low as possible (30.5 $\mu \mathrm{L})$. The composition of these samples is reported in Table 1.

\section{Table 1}

Experimental conditions employed in the present work. $T$ stands for the temperature and $x_{\mathrm{TEA}}, x_{\mathrm{H}_{2} \mathrm{O}}, x_{\mathrm{D}_{2} \mathrm{O}}$ are the molar fractions of TEA, water and deuterium oxide, respectively. Numbers in parentheses stand for the uncertainties.

\begin{tabular}{cccc}
\hline $\begin{array}{c}T / \mathrm{K} \\
( \pm 0.1)\end{array}$ & $\begin{array}{c}x_{\mathrm{TEA}} / \\
\mathrm{mol}^{\mathrm{mol}}{ }^{-1} \\
( \pm 0.0001)\end{array}$ & $\begin{array}{c}x_{\mathrm{H}_{2} \mathrm{O}} / \\
\mathrm{mol} . \mathrm{mol}^{-1} \\
( \pm 0.0001)\end{array}$ & $\begin{array}{c}x_{\mathrm{D}_{2} \mathrm{O}} / \\
\mathrm{mol} \cdot \mathrm{mol}^{-1} \\
( \pm 0.0001)\end{array}$ \\
\hline 283.2 & 0.1149 & 0.7799 & 0.1052 \\
283.2 & 0.1127 & 0.7881 & 0.0992 \\
278.2 & 0.1168 & 0.7825 & 0.1007 \\
278.2 & 0.1167 & 0.7802 & 0.1031
\end{tabular}

The influence of the isotopic substitution of $\mathrm{H}_{2} \mathrm{O}$ with $\mathrm{D}_{2} \mathrm{O}$ has previously been studied for this mixture ${ }^{31}$. Its lower critical solution temperature (LCST) is reduced by $3.80 \mathrm{~K}$ in case of complete substitution. On the other hand, it was found that the transport properties, such as the Fick diffusion coefficient, are independent of the degree of deuteration ${ }^{32}$.

The amount of each component was carefully chosen to place the interface between the two phases under LLE as close as possible to the center of the NMR coil $\left(z_{c c}\right)$, i.e. approximately $2 \mathrm{~cm}$ above the bottom of the NMR tube, cf. Fig. 1A. Once prepared, the samples were allowed to rest for at least 12 hours at ambient temperature and pressure.

\section{Spatially selective sampling}

The experiments were carried out with a $400 \mathrm{MHz}$ Bruker Avance II NMR spectrometer, equipped with a double channel $5 \mathrm{~mm}$ probe (BBO) and triple axis gradients. A robust temperature control $( \pm 0.1$ K) was achieved with a BCU1 unit (Bruker, Rheinstetten) and high quality $5 \mathrm{~mm}$ NMR tubes were used as sampling cell.

A Double Pulsed Field Gradient Selective Echo (DPFGSE) pulse sequence was used to measure the composition distribution along the $z$ coordinate because it is suitable for inhomogeneous systems ${ }^{33}$, cf. Fig 1A. The excitation pulse frequency was set to $22.2 \mathrm{kHz}$. A Gaussian-shaped pulse of $1 \mathrm{~ms}$ and an encoding gradient of 10.61 $\mathrm{G} / \mathrm{cm}$ were used in the gradient echo. The offset $\Omega_{i}$ of the selective pulse was varied between $-20 \mathrm{kHz}$ to $24 \mathrm{kHz}$, which selects horizontal slices or isochromats located at $1.53 \mathrm{~cm}$ and $2.53 \mathrm{~cm}$. The thickness of the isochromat is defined by the bandwidth of the pulse and was determined to be $0.5 \mathrm{~mm}$.

Once the mixing process was established in the NMR tube, spatially selective sampling was performed to map the concentration profile along the $z$ direction. Each mapping consisted of measuring 23 slices along $z$ in random order, an operation that was achieved in $244 \mathrm{~s}$. For each sample according to Table 1, a total of 83 mappings was recorded every 5 minutes for a total duration of 7 hours. The 23 isochromats were chosen to cover a region of $1.1 \mathrm{~cm}$ located inside the NMR coil active region. The acquisition time of the free induction decay (FID) was set to $1 \mathrm{~s}$, and the width of the spectral window was set to $15 \mathrm{ppm}$. The resulting $32 \mathrm{k}$ complex points were stored in a matrix of dimension $83 \times(23 \times 32 \mathrm{k})$. Further data processing, such as apodization $(3 \mathrm{~Hz})$ and baseline correction, was performed using Topspin 2.5 (Bruker Rheinstetten), while integration of the signals was done with in-house scripts written for Scilab 5.5.

\section{Calibration of the spatial coordinate and determination of the bulk temperature}

To precisely estimate the location $z$ of the selected isochromats, it is essential to determine the strength of the pulsed field gradient $G_{z}$ with a good accuracy, cf. supplementary material. This was achieved by calibration with solvents of well-known self-diffusion coefficients. For that purpose, $\mathrm{D}_{2} \mathrm{O}$ with a purity of $99.9 \%$ (Sigma-Aldrich) was used. Transport phenomena in liquid systems are strongly temperature dependent, thus the experimental setup must control this property. Deuterated Methanol (methanol-d4) with a purity of 99.8\% (Sigma-Aldrich) was used to accurately calibrate the bulk temperature measurement, cf. supplementary material.

\section{Extraction of molar fractions}

The intensity $I_{j}$ of the signal was obtained by integrating the signal area and is related to the number of molecules $n_{j}$ present in the sample by ${ }^{34}$

$$
I_{j}=K A_{j} n_{j}
$$

Therein, $K$ is a constant that depends on the experimental setup and $A_{j}$ is the number of protons involved. The integral of the signal was preferred over the signal height because it is less dependent on the individual spin relaxation rates, cf. supplementary material. It is possible to demonstrate that the molar fraction $x_{j}$ is readily obtained from the intensities by

$$
x_{j}=\frac{A_{j}^{-1} I_{j}}{\sum_{k=1}^{n} A_{k}^{-1} I_{k}} .
$$

In the present experiments, the relaxation rates depend on time and spatial location. Therefore, a longer relaxation time of $4 \mathrm{~s}$ was chosen to ensure that all spins had relaxed. Finally, the accumulation of only two acquisitions was necessary to obtain a high signal-to-noise ratio. 
A

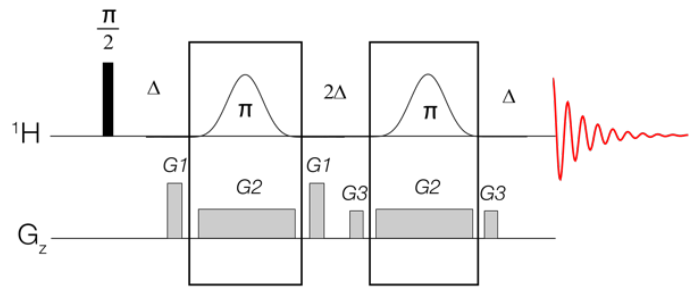

B

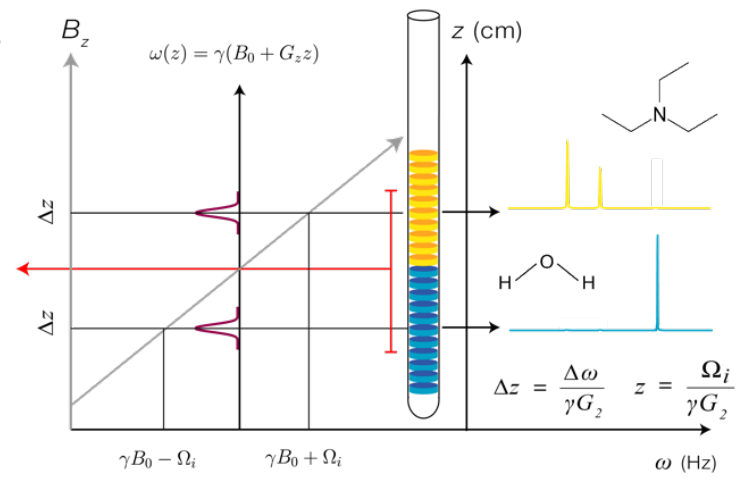

Figure 1. A) Double Pulsed Field Gradient Selective Echo (DPFGSE) pulse sequence ${ }^{33}$. NMR signals can be spatially encoded by applying a pulse field gradient simultaneously with a narrowband rf-pulse ${ }^{35}$. B) The field gradient shifted the Larmor frequency linearly in the $z$ coordinate, while the narrow band pulse ${ }^{36}$ was used to select the vertical region of interest by tuning its resonance frequency offset $\Omega_{i}$. The resonance frequency at this $z$ coordinate is thus defined as shown in panel $B$ ), where $\omega_{z}(z)$ represents the resonance frequency of the nuclei with gyromagnetic constant $\gamma, B_{0}$ is the magnitude of the external magnetic field and $G_{z}$ the strength of the gradient at coordinate $z$. The thickness of the selected slice is directly related to the bandwidth of pulse. Repeating the experiment by varying the frequency offset of the selective pulse allows for the observation of the composition the different coordinates $z$.

\section{Measuring a gradient in the NMR tube}

TEA/ $\mathrm{H}_{2} \mathrm{O}$ exhibits a LLE region with a LCST, i.e., starting from a twophase state point, the temperature has to be decreased to enter the homogeneous region where mixing occurs, cf. Fig. 2B (insert). Therefore, to establish and measure a gradient of concentration in the NMR tube, the system is first set to equilibrate a temperature where both phase coexist $\left(T_{\text {initial }}=300 \mathrm{~K}\right)$. Subsequently, this system was cooled below the mixing temperature $\left(T_{\text {mixing }}=293.7 \mathrm{~K}\right)$ to a final temperature $\left(T_{o b s}=278,2 \mathrm{~K}\right.$ or $\left.283.2 \mathrm{~K}\right)$, as described in Figure 2B (insert). While reaching its new equilibrium, sampling of the concentration gradient were recorded as described above.

Prior to the experiment, the concentration profile was measured to locate the position of the interface separating the two phases under LLE, which was near the center of the NMR coil, cf. Figure 2A. Before acquiring data, a time delay $\boldsymbol{t}_{\text {obs }}$ (Figure 2B) was necessary to avoid the sampling of contributions from convective phenomena presents instep 1 and 2. The measurements takes place during step 3 when the remaining flux $\boldsymbol{N}_{\boldsymbol{i}}$ in the tube was only caused by diffusive contributions $\boldsymbol{J}_{\boldsymbol{i}}$. This process was repeated for all samples listed in Table 1.
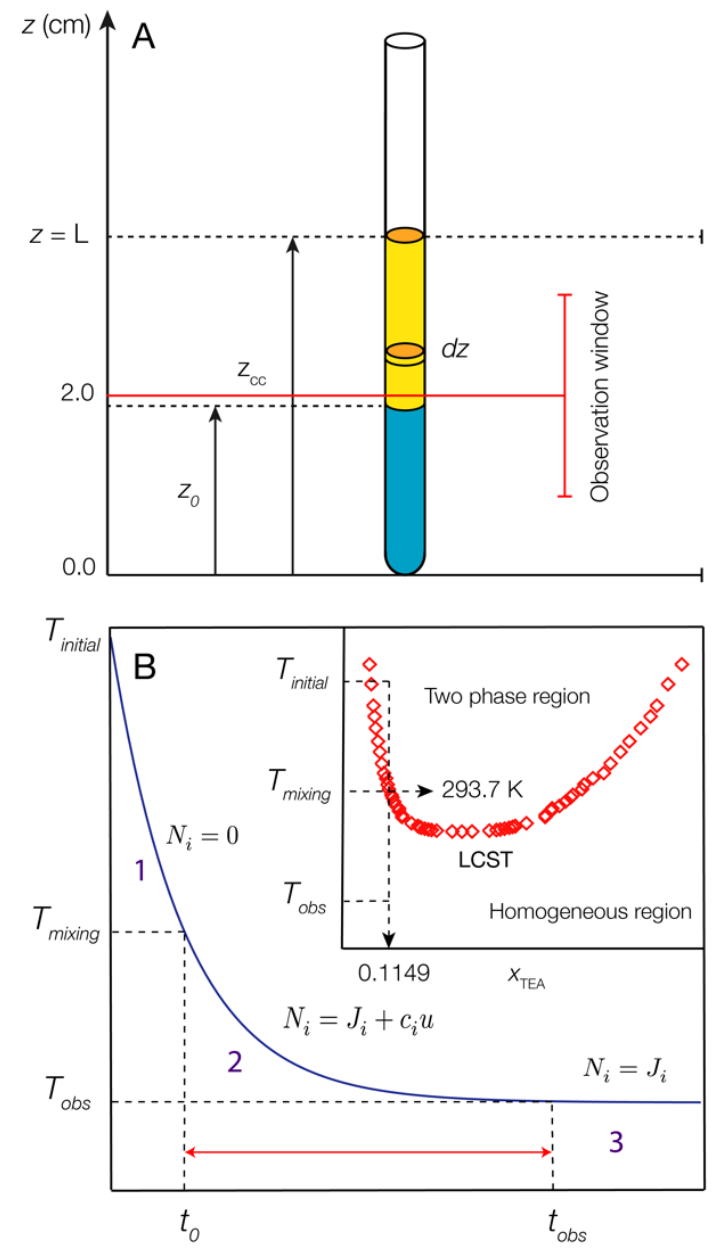

Figure 2. The experimental setup to observe the time $t$ evolution of the molar fractions $x_{i}(z, t)$ during the mixing process, where $z$ is the relevant spatial coordinate $A$ ) The sampled sections were chosen to be near the center of the observation window (red vertical line), defined by the height of the coil in the NMR setup (not depicted). Experimentally, this window is determined as the largest portion of the coil for which the response is homogeneous, while the intensity of the signal is reduced at the edges of the coil. The preparation of the sample ensured that the LLE interface was at the center of the observation window, i.e., about $2 \mathrm{~cm}$ above the bottom of the NMR tube in the present setup. B) Cooling curve and phase diagram (insert) of the TEA $/ \mathrm{H}_{2} \mathrm{O}$ system ${ }^{37}$. The measurement cycle consisted of preparing the sample at a temperature where two phases coexist $T_{\text {initial }}$, cool it down below $T_{\text {mixing }}$ to a target temperature $T_{o b s}$ in the homogeneous region, and sample how this new equilibrium is attained. The mixing temperature is defined as the crossing point in the liquid-liquid coexistence curve (red diamonds) for the composition of our system $\left(X_{T E A}=0.1149\right)$. During cooling, diffusive $J_{i}$ and convective contributions to the flux are present (steps 1 and 2). Measurements started after a temporal delay that ensured that contributions from convective processes had subsided (step 3). 


\section{Modeling the mixing process in an NMR tube}

The assumption of constant volume is not valid for the binary mixture TEA/ $\mathrm{H}_{2} \mathrm{O}$, which exhibits a significant excess volume $v^{E}\left(T, p, x_{1}\right)$ at $283.15 \mathrm{~K}^{38}$ and $278.15 \mathrm{~K}^{39}$. An appropriate mass transfer model was proposed by Bardow et al. ${ }^{23}$ employing a coordinate transformation proposed in Ref. ${ }^{40}$. The continuity equation is solved in a molar reference frame because the total number of moles is constant during the diffusion process. However, this reference frame is defined using a transformed concentration $\xi_{i}^{M}=x_{i} / v_{n}^{0}$, where $v_{n}^{0}$ is the molar volume of a pure reference component. In this new reference frame, the continuity equation of component 1 in the binary mixture can be recast as

$$
\frac{\partial \xi_{1}^{M}}{\partial t}=\frac{\partial}{\partial \bar{z}}\left[D_{12}\left(c_{t} v_{1}^{0}\right)^{2} \frac{\partial \xi_{1}^{M}}{\partial \bar{z}}\right]
$$

Therein, $v_{1}^{0}(T, p)$ is the molar volume of TEA, $D_{12}$ the Fick diffusion coefficient, $z$ the laboratory framework coordinate and $\bar{z}$ represents a fictional distance which is related to $z$ through ${ }^{23}$

$$
\bar{z}=\int_{0}^{z} c_{t}\left(t, z^{\prime}\right) v_{n}^{0} d z^{\prime}
$$

Furthermore, the total concentration is given by $1 / c_{t}=$ $\sum_{i} x_{i} v_{i}^{0}(T, p)+v^{E}\left(T, p, x_{1}\right)$. For the excess volume, a Redlich-Kister type correlation was fitted to experimental data

$$
\begin{aligned}
& v^{E}\left(T, p, x_{1}\right)=x_{1}\left(1-x_{1}\right)\left[A+B\left(2 x_{1}-1\right)+\right. \\
& \left.C\left(2 x_{1}-1\right)^{2}+D\left(2 x_{1}-1\right)^{3}\right] .
\end{aligned}
$$

which excellently describes the experimental literature data ${ }^{38}{ }^{39}$, cf. Fig. 3C, D. Coefficients for both target temperatures are reported in Table $1 S$ of the supplementary material. The effect of $\mathrm{D}_{2} \mathrm{O}$ as a third component on the excess properties has been well studied and is expected to be small for compositions where its molar fraction is below $0.999^{41}$.

\section{Results and discussion}

To illustrate how the present approach may be used to access the composition dependence of the Fick diffusion coefficient of a binary mixture, TEA $/ \mathrm{H}_{2} \mathrm{O}$ systems were prepared as described above. A given sample was cooled down below the temperature of mixing and, after a temporal delay that allowed the convective flux to vanish, the concentration profile along the $z$ coordinate was measured over time. Fig. 3 shows the first sampled concentration profile that was used as initial condition for TEA in the new coordinate system. A strategy to solve the resulting PDE is outlined in the following.

\section{Initial condition}

Although the mixing process occurs inside the NMR magnet, it is not possible to start the measurement immediately, and a temporal delay is necessary to ensure that contributions from convective processes have terminated, i.e., the temperature had to attain a constant value. Here, this condition was chosen to initiate the first mapping at $t_{o b s}, \mathrm{cf}$. Figure 2B. This issue is not specific to the present method and the effects of such a choice have been discussed for other experimental techniques ${ }^{42}$. An error function was used to fit the initial condition in the new reference frame at $t=t_{\text {obs }}$.

$$
\xi\left(\bar{z}, t=t_{o b s}\right)=U+\operatorname{Verf}[Y(z+W)] .
$$

Therein, $\boldsymbol{U}, \boldsymbol{V}, \boldsymbol{Y}$, and $\boldsymbol{W}$ are fitting parameters as reported in Table $1 \mathrm{~S}$ of the supplementary material. Because the observation window was limited by the length of the active volume of the coil, the concentration profile of the mixture beyond these limits was extrapolated. Fig. 3 shows the initial condition in the new reference frame for both target temperatures. It allows for the definition of boundary conditions that are required to solve Eq. (3).
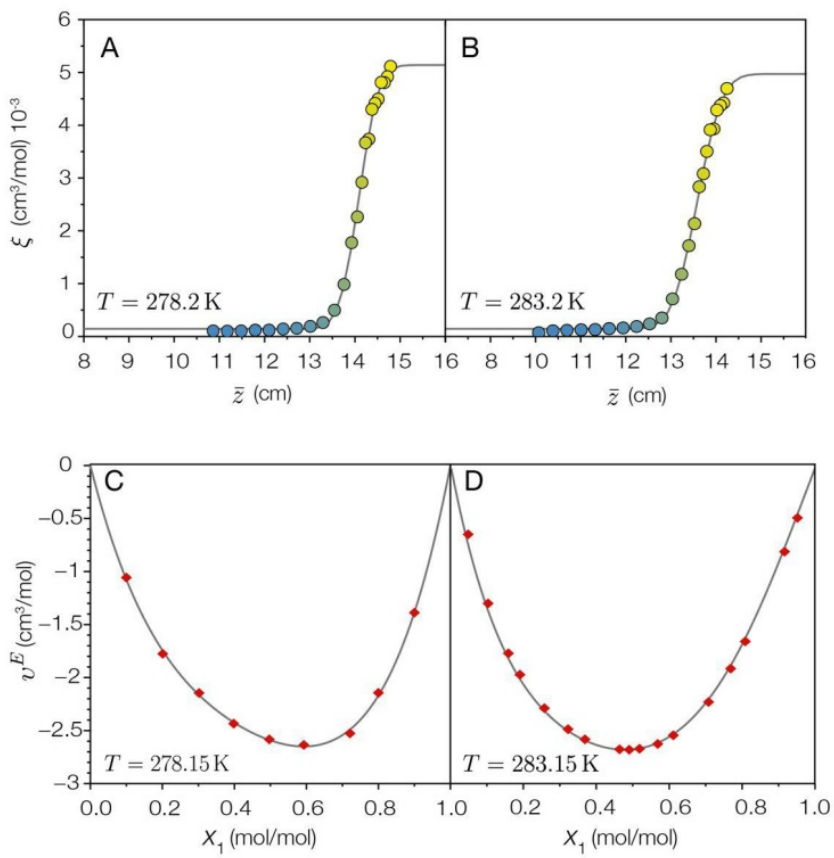

Figure 3. Initial condition $\xi(\bar{z}, 0)$ for solving Eq. (3) for temperatures $278.2 \mathrm{~K}$ and $283.2 \mathrm{~K}$ as well as excess volume $v^{E}\left(T, p, x_{1}\right)$. The initial condition was built using the fictional distance $\bar{z}$ obtained by means of Eq. (6) and a new composition variable calculated using molar volume $v_{1}^{0}(T, p)$ for TEA. The experimental data (A) and (B) can be approximated with the error function. The parameters calculated by least squares are presented for each temperature. Note that the separation between the points is not regularly spaced because the fictional distance is proportional to $c_{t}$ at specific locations. The four parameter Redlich-Kister model was fitted to experimental excess volume data (C) and (D) from the literature ${ }^{38} 39$. Negative excess volume values reflect contraction of the liquid during mixing.

\section{Numerical solution}

The PDE, Eq. (3), was solved numerically for TEA/ $\mathrm{H}_{2} \mathrm{O}$ with the method of the lines ${ }^{43}$ as implemented in the Mathematica software. The fictional distance $\bar{z}$ was chosen as the variable to be discretized. The composition dependence of Fick diffusion coefficient can be represented by a polynomial ${ }^{44}$

$$
D_{12}\left(T, p, x_{1}\right)=\sum_{k=1}^{N} v_{k} x_{1}^{k-1} \text {. }
$$


Therein, $x_{1}$ is the molar fraction of component 1 and $v_{k}$ are fitting parameters. In case of the mixture Toluene/Cyclohexane, a combination of low order polynomials was sufficient to describe the Fick diffusion coefficient in the entire composition range ${ }^{45}$. Here, the following functionality was deduced by adjustment to present experimental data

$$
D_{12}\left(T, p, x_{1}\right)=v_{4} x_{1}^{3}+v_{2}\left(x_{1}-x_{1}^{2}\right) .
$$

This expression was transformed in terms of the new composition variable $\xi$ and inserted into Eq. (3). Once the PDE was solved in the new coordinate space $(\xi, \bar{z})$, the results were converted back to the laboratory reference frame by reversing the expression $\xi_{i}^{M}=x_{i} / v_{n}^{0}$

$$
\begin{gathered}
z=\frac{1}{v_{1}^{0}(T, p)} \int_{0}^{\bar{z}}\left[x_{1} v_{1}^{0}(T, p)+\left(1-x_{1}\right) v_{2}^{0}(T, p)\right. \\
\left.+v^{E}\left(T, p, x_{1}\right)\right] d \bar{z} .
\end{gathered}
$$

Solutions for specific combinations of the coefficients $v_{2}$ and $v_{4}$ were parametrized to maximize the correlation coefficient $R^{2}$. The results obtained for the target temperatures $278.15 \mathrm{~K}$ and $283.15 \mathrm{~K}$ are shown in Fig. 4 and reported numerically in Table $2 \mathrm{~S}$ of the supplementary material.

\section{Comparison to other data}

Predictive models often assume a relationship between the propagation of molecular species quantified by the intra-diffusion coefficients $D_{i}^{*}$ and the Fick diffusion coefficient $D_{12}$. The Darken equation ${ }^{46}$ is particularly straightforward

$$
D_{12}=\left(x_{2} D_{1}^{*}+x_{1} D_{2}^{*}\right) .
$$

This expression can be meaningful if the involved components exhibit similar intermolecular interactions between like and unlike species, as is the case for some metal alloys ${ }^{46}$ or ideal mixtures ${ }^{47}$. However, once the behavior of mixtures is associated with nonideality, it is necessary to consider the thermodynamic factor [ $1+$ $\left.\partial \ln \gamma_{1} / \partial x_{1}\right]^{48}$

$$
D_{12}=\left(x_{2} D_{1}^{*}+x_{1} D_{2}^{*}\right)\left[1+\frac{\partial \ln \gamma_{1}}{\partial x_{1}}\right]
$$

The thermodynamic factor is usually extracted from vapor-liquid equilibrium (VLE) data. D'Agostino et al. ${ }^{28}$ calculated this factor for the TEA $/ \mathrm{H}_{2} \mathrm{O}$ mixture using experimental VLE data reported by Counsell ${ }^{49}$. Modifications to Eq. (11) were recently presented for binary mixtures that have a consulate point, such as the Hexane/Nitrobenzene $e^{50}$ or for non ideal mixtures ${ }^{51}$. Given its success in predicting $D_{12}$ over a wide composition range, a similar modification was proposed for $\mathrm{TEA} / \mathrm{H}_{2} \mathrm{O}$ in the vicinity of its consulate point ${ }^{28}$

$$
D_{12}=\left(x_{2} D_{1}^{*}+2 x_{1} D_{2}^{*}\right)\left[1+\frac{\partial \ln \gamma_{1}}{\partial x_{1}}\right]^{\alpha} \text {. }
$$

Theoretical considerations have been presented to motivate the exponent $\alpha^{52}$. However, it can also be seen as a fitting parameter to alleviate shortcomings of the Darken equation which is more closely related to the Maxwell-Stefan diffusion coefficient.
The present results obtained by solving the PDE are contrasted in Fig. 5 with numerical models and experimental literature data ${ }^{29}{ }^{30}$. For the sake of comparison, we applied our methodology at temperatures for which Fick diffusion coefficient data for TEA/ $\mathrm{H}_{2} \mathrm{O}$ are available in the literature.
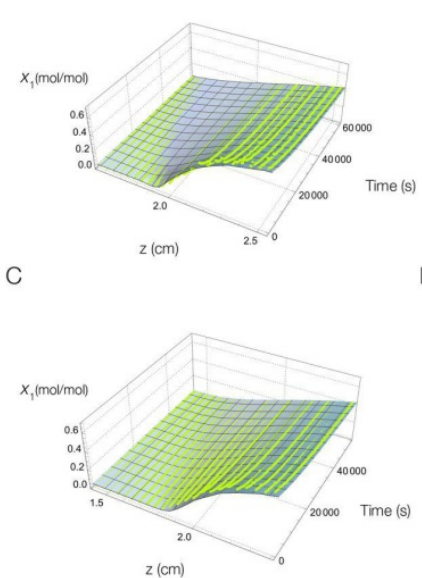
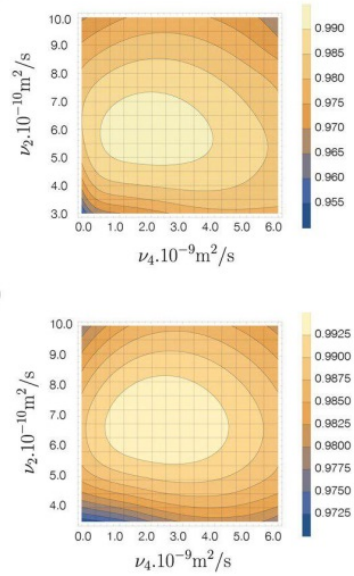

Figure 4. Experimental data measured at $278.2 \mathrm{~K}(\mathrm{~A})$ and $283.2 \mathrm{~K}(\mathrm{C})$ are contrasted with the numerical solution of the PDE for each case. The spatial variable was discretized by 500 points between $1.4 \mathrm{~cm}$ and $2.8 \mathrm{~cm}$. Parameters $v_{2}$ and $v_{4}$ were fitted to maximize the correlation coefficient $R^{2}$ (B and D).

Both predictive models achieve a better match with the experimental literature data than the present approach, in particular for low TEA molar fractions $\left(x_{1}<0.2 \mathrm{~mol} / \mathrm{mol}\right)$. However, when the entire composition range is considered, the present results are equally consistent with the experimental literature data. For a fair assessment, the following issues have to be considered:

1. The predictive models are more accurate, but they require a large amount of experimental transport data as an input because they rest on intra-diffusion coefficients of both components over the entire composition range.

2. The predictive models also rely on the thermodynamic factor that may greatly vary depending on the choice of the activity coefficient mode ${ }^{53}$ and requires information on the VLE.

It must be returned to the central idea of the method first proposed by Gupta and Cooper ${ }^{17}$ to understand the discrepancies observed for diluted states, cf. Supplementary material. Instead of carrying out several experiments varying the molar fraction, its time evolution is followed by a single experiment. In this approach, data about low (or high) TEA molar fractions may only be captured during the very sharp gradients present immediately after the mixing occurs (or after infinite time). Because of convective phenomena present at the beginning of the mixing process it is not possible to record an infinitely sharp gradient, nor it is possible to continue the experiment until any gradient at all.. For instance, our experiment starts from a composition dictated by the LLE of the system $\left(x_{1}=0.75 \mathrm{~mol} / \mathrm{mol}\right)$ and stops before perfect equilibrium is attained. Given these conditions, some degree of uncertainty is unavoidable in dilute regimes. This problem has already been observed in the implementation of an incremental model for Ethyl acetate/Cyclohexane ${ }^{26}$ and its explanation is consistent with our 
results; deviations observed between the two repetitions are larger for molar fractions near infinite dilution. The use of an a priori model when solving the PDE has been discussed elsewhere ${ }^{19} 20$. Ideally, one would like to discover an appropriate model from the experimental data only.
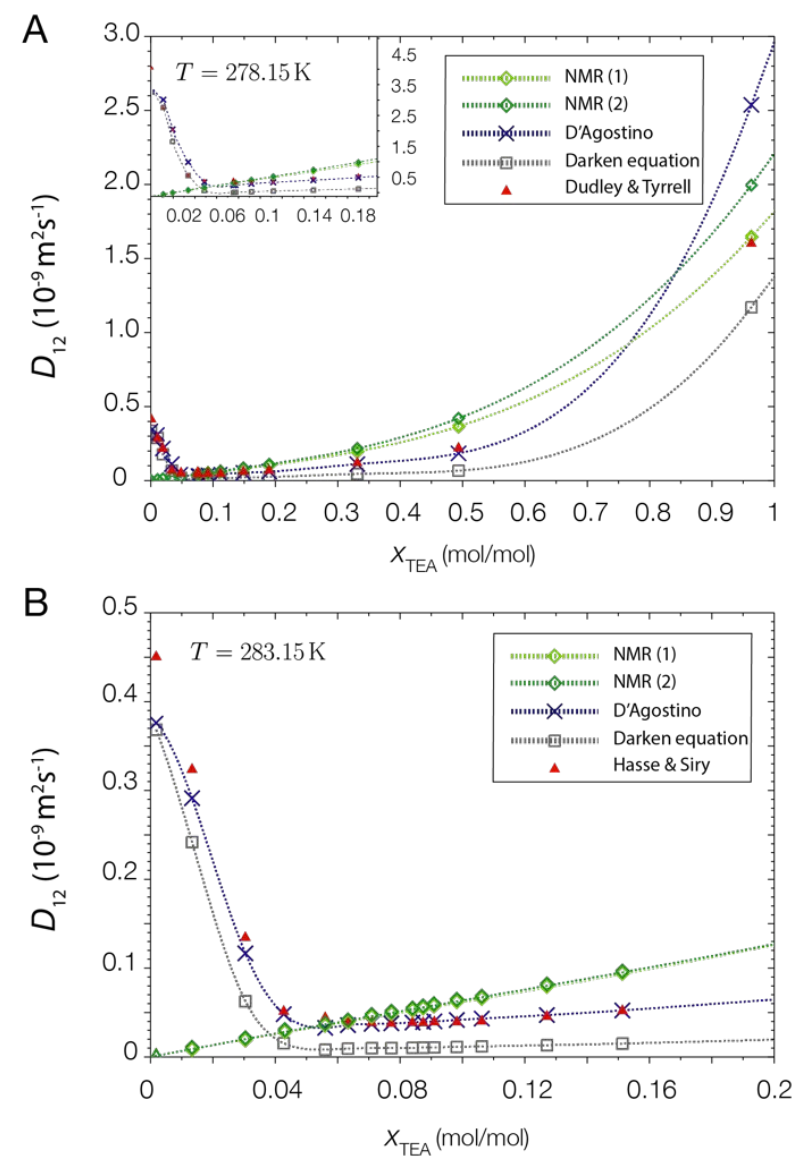

Figure 5. Fick diffusion coefficient obtained by solving the PDE for TEA $/ \mathrm{H}_{2} \mathrm{O}$ in comparison to experimental literature data by Dudley and Tyrell at $278.15 \mathrm{~K}^{29} \mathrm{~A}$ ) and $283.15 \mathrm{~K}^{30} \mathrm{~B}$ ), interpolating the data for the latter. The predictive models of Darken and D'Agostino et al. were applied by using intra-diffusion coefficients and thermodynamic factor data reported in Ref. ${ }^{28}$. The green lines represent the Fick diffusion coefficient obtained by NMR. The dark and light green lines represent the first and second replicate, following the order in Table 1.

Indeed, such an approach has been successfully applied in recent work using Raman spectroscopy ${ }^{54}$. It is assumed that the same method, translated to our work, may deliver appropriate models and accurate diffusion coefficients, provided that it is supported by a suitable experimental design. For instance, a better understanding of the impact of the initial cooling period is crucial because it affects several factors, such as the position of the center and shape of the first measured gradient. Further experiments in this direction are currently under development.

\section{Conclusions}

A complementary strategy is presented for the determination of the Fick diffusion coefficient in a wide composition range for non-ideal systems. This approach is nevertheless limited to systems that possess an LCST or an upper critical solution temperature (UCST), as a mean to establish a reproducible gradient inside of an NMR tube. Even with the strong restrictions imposed by the NMR tube, different experimental designs may, in the future, extend this method to other systems without consolute point. The formulated PDE for the binary mixture TEA/Water was solved numerically, adopting a reduced functionality to estimate the binary Fick diffusion coefficient $D_{12}$ through fitting to NMR data. The results are consistent with experimental literature data measured with other techniques. Predictive models developed for the TEA/ $\mathrm{H}_{2} \mathrm{O}$ system were also compared with the functionality obtained for the Fick diffusion coefficient.

Despite the restrictions discussed above, the reduction in experimental effort is notable, which may compensate for a larger uncertainty in dilute regimes. Considering the spectroscopic benefits to be comparable to those provided by Raman, the NMR setup allows for the measurement at different temperatures out-of-the-box, which is essential for a broader study of mass transport phenomena.

\section{Conflicts of interest}

There are no conflicts to declare.

\section{Acknowledgements}

The Paderborn chair gratefully acknowledges financial support by Deutsche Forschungsgemeinschaft (grant VR6/11).

\section{References}

E. L. Cussler, Diffusion: Mass Transfer in Liquids, Cambridge University Press, Cambridge, 1997. S. Jariwala and B. Krishnamurthy, Transport equations in an enzymatic glucose fuel cell, Chem. Phys. Lett., 2018, 692, 7. J. Yuan and B. Sundén, On mechanisms and models of multicomponent gas diffusion in porous structures of fuel cell electrodes, Int. J. Heat Mass Transf., 2014, 69, 358.

P. J. Dunlop, K. R. Harris, D. J. Young, B. W. Rossiter and R. C. Baetzold, Physical Methods of Chemistry, Wiley, New York, 1992.

J. W. Mutoru and A. Firoozabadi, Form of multicomponent Fickian diffusion coefficients matrix, J. Chem. Thermodyn., 2011, 43, 1192.

Y. M. Muñoz-Muñoz, G. Guevara-Carrion, M. LlanoRestrepo and J. Vrabec, Lennard-Jones force field parameters for cyclic alkanes from cyclopropane to cyclohexane, Fluid Phase Equilib., 2015, 404, 150.

G. Guevara-Carrion, Y. Gaponenko, T. Janzen, J. Vrabec and V. Shevtsova, Diffusion in Multicomponent Liquids: From Microscopic to Macroscopic Scales, J. Phys. Chem. B, 2016, 120, 12193.

R. Taylor and R. Krishna, Multicomponent Mass Transfer, John Wiley \& Sons, New York, 1993.
R. Krishna and J. A. Wesselingh, The Maxwell-Stefan approach to mass transfer, Chem. Eng. Sci., 1997, 52, 861. M.-Á. Gómez-García, I. Dobrosz-Gómez, E. GilPavas and J. 
Rynkowski, Simulation of an industrial adiabatic multi-bed catalytic reactor for sulfur dioxide oxidation using the Maxwell-Stefan model, Chem. Eng. J., 2015, 282, 101.

A. Kubaczka, W. Kamiński and J. Marszałekb, Predicting mass fluxes in the pervaporation process using MaxwellStefan diffusion coefficients, J. Memb. Sci., 2018, 546, 111.

G. Guevara-Carrion, T. Janzen, Y. M. Muñoz-Muñoz and J. Vrabec, Mutual diffusion of binary liquid mixtures containing methanol, ethanol, acetone, benzene, cyclohexane, toluene, and carbon tetrachloride, J. Chem. Phys., 2016, 144, 124501.

D. G. Leaist, Determination of ternary diffusion coefficients by the Taylor dispersion method, J. Phys. Chem., 1990, 94, 5180.

D. G. Miller, The History of Interferometry for Measuring Diffusion Coefficients, J. Solution Chem., 2014, 43, 6.

T. Janzen, S. Zhang, A. Mialdun, G. Guevara-Carrion, J. Vrabec, M. He and V. Shevtsova, Mutual diffusion governed by kinetics and thermodynamics in the partially miscible mixture methanol + cyclohexane, Phys. Chem. Chem. Phys., 2017, 19, 31856-31873.

16 R. C. Reid, J. M. Prausnitz and B. E. Poling, The Properties of Gases and Liquids, McGraw-Hill, New York, 1987.

17 P. K. Gupta and A. R. Cooper, The [D] matrix for multicomponent diffusion, Physica, 1971, 54, 39.

A. Bardow, W. Marquardt, V. Göke, H.-J. Koss and K. Lucas, Model-based measurement of diffusion using Raman spectroscopy, AlChE J., 2003, 49, 323.

G. Arvind and S. K. Bhatia, Determination of concentrationdependent adsorbate diffusivities by numerical inversion, Chem. Eng. Sci., 1995, 50, 1361.

20 R. K. Lewus and G. Carta, Protein Transport in Constrained Anionic Hydrogels: Diffusion and Boundary-Layer Mass Transfer, Ind. Eng. Chem. Res., 2001, 40, 1548.

21 A. T. Wagner and H.-H. Kohler, Determination of the concentration dependence of polyelectrolyte diffusion coefficients by application of the Boltzmann gradient method, J. Colloid Interface Sci., 2008, 319, 555.

A. Bardow and W. Marquardt, Identification of diffusive transport by means of an incremental approach, Comput. Chem. Eng., 2004, 28, 585.

A. Bardow, V. Göke, H.-J. Koß, K. Lucas and W. Marquardt, Concentration-dependent diffusion coefficients from a single experiment using model-based Raman spectroscopy, Fluid Phase Equilib., 2005, 357.

P. C. Lauterbur, Image Formation by Induced Local Interactions: Examples Employing Nuclear Magnetic Resonance, Nature, 1973, 242, 190.

A. Bardow, E. Kriesten, A. M. Voda, F. Casanova, B. Blümich and W. Marquardt, Prediction of multicomponent mutual diffusion in liquids: Model discrimination using NMR data, Fluid Phase Equilib., 2009, 278, 27.

E. Kriesten, M. A. Voda, A. Bardow, V. Göke, F. Casanova, B. Blümich, H.-J. Koss and W. Marquardt, Direct determination of the concentration dependence of diffusivities using combined model-based Raman and NMR experiments, Fluid Phase Equilib., 2009, 277, 96.
C. F. Pantoja, J. A. Bolaños, A. Bernal and J. Wist, Mutual Diffusion Driven NMR: a new approach for the analysis of mixtures by spatially resolved NMR spectroscopy, Magn. Reson. Chem., 2017, 55, 519.

C. D'Agostino, M. D. Mantle, L. F. Gladden and G. D. Moggridge, Prediction of mutual diffusion coefficients in non-ideal mixtures from pulsed field gradient NMR data: Triethylamine-water near its consolute point, Chem. Eng. Sci., 2012, 74, 105.

29 G. J. Dudley and V. H. J. Tyrrel, Transport processes in binary and ternary mixtures containing water, triethylamine and urea. Part 2.-Mutual diffusion coefficients in the triethylamine + water system at 5 and $15^{\circ} \mathrm{C}, \mathrm{J}$. Chem. Soc. Faraday Trans. 1 Phys. Chem. Condens. Phases, 1989, 69, 2200.

30 R. Haase and M. Siry, Diffusion im kritischen Entmischungsgebiet binärer flüssiger Systeme, Zeitschrift für Phys. Chemie, 1968, 57, 56.

31 D. V. Fenby, Z. S. Kooner and J. R. Khurma, Deuterium isotope effects in liquid-liquid phase diagrams: A review, Fluid Phase Equilib., 1981, 7, 327.

G. Wittko and W. Köhler, Influence of isotopic substitution on the diffusion and thermal diffusion coefficient of binary liquids, Eur. Phys. J. E, 2006, 21, 283.

W. Kozminski, Application of spatially resolved NMR spectroscopy for high resolution of heterogeneous samples, Pol. J. Chem., 2000, 74, 1185m.

34 F. Malz and H. Jancke, Validation of quantitative, J. Pharm. Biomed. Anal., 2005, 38, 813.

B. Shapira and L. Frydman, Spatial Encoding and the Acquisition of High-Resolution NMR Spectra in Inhomogeneous Magnetic Fields, J. Am. Chem. Soc., 2004, 126, 7184.

H. Geen and R. Freeman, Band-selective radiofrequency pulses, J. Magn. Reson., 1991, 93, 93.

37 R. Gastaud and D. Beysens, The Lorentz-Lorenz relationship in the phase diagram of triethylamine and water. Application to the coexistence curve, J. Chem. Phys., 1990, 93, 3432--3436.

T. M. Letcher and W. Spiteri, The excess volume for triethylamine + water, J. Chem. Thermodyn., 1979, 11, 905. E. F. Zhuravlev, Russian Journal of General Chemistry, Russ. J. Gen. Chem., 1961, 31, 363.

40 J. Crank, The Mathematics of Diffusion, Clarendon Press, Oxford, 1975.

G. Jancsó, H2O-D2O Solvent Isotope Effect on Excess Molar Volumes of 3-Methylpyridine Solutions, J. Solution Chem., 2006, 35, 991.

A. Bardow, Ph.D. thesis RWTH Aachen, 2004.

43 W. E. Schiesser, The Numerical Method of Lines: Integration of Partial Differential Equations, Elsevier Science, San Diego, 2012.

P. Duchateau, Monotonicity and Uniqueness Results in Identifying an Unknown Coefficient in a Nonlinear Diffusion Equation, SIAM J. Appl. Math., 1981, 41, 310--323. coefficients and densities for binary organic liquid mixtures, 
J. Chem. Data, 1971, 16, 424. through Free Energy in binary metallic Systems, Trans. AIME, 1948, 175, 184.

47 W. Marbach, H. G. Hertz and H. Weingärtner, Self- and Mutual Diffusion Coefficients of some Binary Liquid $n$ Alkane Mixtures - A Velocity Correlation Study -, Zeitschrift für Phys. Chemie, 1995, 189, 63.

48 N. Karpe, J. P. Krog, J. Bøttiger, N. G. Chechenin, R. E. Somekh and A. L. Greer, The thermodynamic factor in interdiffusion: A strong effect in amorphous Ni-Zr, Acta Metall. Mater., 1995, 43, 551.

49 J. F. Counsell, University of Bristol, 1959.

50 C. D'Agostino, M. D. Mantle, L. F. Gladden and G. D. Moggridge, Prediction of binary diffusion coefficients in non-ideal mixtures from NMR data: hexane- nitrobenzene near its consolute point, Chem. Eng. Sci., 2011, 66, 3898.

51 Q. Zhu, G. D. Moggridge and C. D'Agostino, A local composition model for the prediction of mutual diffusion coefficients in binary liquid mixtures from tracer diffusion coefficients, Chem. Eng. Sci., 2015, 132, 250-258.

52 E. L. Cussler, Cluster diffusion in liquids, AIChE J., 1980, 26, 43.

53 R. Taylor and H. A. Kooijman, Composition derivatives of activity coefficient models (for the estimation of thermodynamic factors in diffusion), Chem. Eng. Commun., 1991, 102, 87.

54 A. Bardow, V. Göke, H.-J. Koß, K. Lucas and W. Marquardt, Concentration-dependent diffusion coefficients from a single experiment using model-based Raman spectroscopy, Fluid Phase Equilib., 2005, 228, 357. 